\title{
Calcium-based versus non-calcium-based phosphate binders: effect on mortality in patients with CKD
}

U se of phosphate binders is recommended for lowering serum phosphate level and treating hyperphosphataemia in patients with chronic kidney disease (CKD); however, the benefit of non-calcium-based versus calcium-based phosphate binders has been a matter of debate. An updated systematic review and meta-analysis now reports that non-calcium-based phosphate binders are associated with a significantly decreased risk of all-cause mortality compared with calcium-based phosphate binders. On the basis of their findings, the investigators suggest that non-calcium-based phosphate binders-specifically sevelamer or lanthanum - should be the first-line therapy for phosphate lowering in patients with CKD.

Hyperphosphataemia-resulting from metabolic disturbances and an inability of the kidneys to excrete excess dietary phosphate-is a common complication of advanced CKD. It is thought to contribute to the excess cardiovascular mortality associated with CKD by promoting the conversion of vascular smooth muscle cells into bone forming, osteoblast-like cells, leading to vascular calcification. Observational studies have demonstrated an association between cardiovascular mortality and increased levels of serum calcium, phosphate, and calciumphosphate product in patients with CKD.

Control of high serum phosphate levels is achieved through dietary phosphate restriction and use of phosphate binders. However, despite an assumption that use of non-calcium-based phosphate binders might slow the development of vascular calcification by reducing both calcium and phosphate levels, a benefit of non-calcium-based binders over the cheaper calcium-based binders has not been definitively demonstrated.

To better understand which type of phosphate binder should be used in patients with CKD, Sophie Jamal and

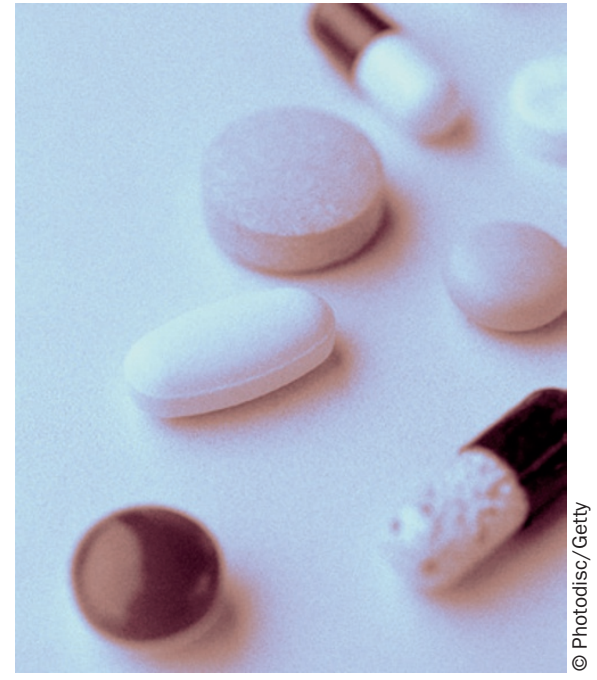

colleagues performed a meta-analysis in 2009 to investigate the effects of calcium-based versus non-calciumbased phosphate binders on mortality in patients with CKD. On the basis of data from eight randomized controlled trials, the researchers identified a nonsignificant trend towards a decrease in all-cause mortality in patients receiving non-calcium-based phosphate binders (sevelamer hydrochloride, sevelamer carbonate, or lanthanum) compared with those receiving calcium-based phosphate binders (calcium carbonate or calcium acetate; risk ratio [RR] 0.68, 95\% CI 0.41$1.11)$. Given the low power of this analysis, the researchers have now performed an updated meta-analysis to include trials published since their original study. "Trials that have been published since our original meta-analysis are inconclusive as to the relation between type of phosphate binder and mortality," explain the researchers. "As such, we aimed to provide the best available evidence as to which type of phosphate binder clinicians should prescribe to patients with chronic kidney disease." The researchers identified eight new studies $(n=3,230)$, including five randomized controlled trials, that met their inclusion criteria and added them to the 10 studies included in their previous analyses.

Analysis of the 11 randomized trials that reported mortality outcomes showed that patients assigned to receive non-calcium-based binders $(n=2,312)$ had a $22 \%$ reduction in all-cause mortality compared with patients assigned to receive calcium-based binders ( $n=2,310$; RR $0.78,95 \%$ CI $0.61-0.98)$. An analysis of nonrandomized trials and a combined analysis of nonrandomized and randomized trials showed similar benefits, with $11 \%$ and $13 \%$ reductions in all-cause mortality associated with use of noncalcium-based binders, respectively.

The reduction in mortality associated with non-calcium-based binders seemed to be independent of the change in serum phosphate concentration, as no significant differences by treatment assignment were reported. However, the researchers noted an increase in coronary artery calcification in patients receiving calcium-based binders compared with those on noncalcium-based binders, suggesting that vascular calcification may have a role in the increased mortality associated with this treatment. Jamal and colleagues say that further research is needed to unequivocally show the effects of phosphate binder type on mortality, to investigate the mechanisms of death in patients on phosphate binders, and to assess whether outcome differs by type of non-calcium-based binder used.

\section{Susan J. Allison}

Original article Jamal, S. A. et al. Effect of calcium-based
versus non-calcium-based phosphate binders on mortality
in patients with chronic kidney disease: an updated
systematic review and meta-analysis. Lancet doi:10.1016/
S0140-6736(13)60897-1
Further reading Jamal, S. A. et al. The effects of calcium-
based versus non-calcium-based phosphate binders on
mortality among patients with chronic kidney disease:
a meta-analysis. Nephrol. Dial. Transplant. 24,3168-3174
(2009)

